\title{
Evaluation of Two Photometers Used in Enzyme-Linked Immunosorbent Assays
}

\author{
V. M. GENTA† AND J. H. BOWDRE* \\ Clinical Microbiology Laboratories, North Carolina Memorial Hospital, University of North Carolina School \\ of Medicine, Chapel Hill, North Carolina 27514
}

Received 27 August 1981/Accepted 13 April 1982

The performance of two commercially available high-speed photometers, designed for through-the-plate reading, was evaluated. Linearity of instrumental reading and reproducibility of same-day and 2-day measurements were assessed by least-squares analysis and analysis of variance, respectively. For both instruments, the photometric error was on the order of thousandths of an absorbance unit and was much smaller than the error of the currently available enzyme-linked immunosorbent assays.

High-speed photometers designed for through-the-plate reading insure a rapid turnover time for measuring colorimetric reactions in microtrays. Consequently, the quality of these instruments is an important consideration in the adoption of enzyme-linked immunosorbent assays (ELISA) and similar assays by laboratories with large numbers of specimens. Unlike conventional photometers, these photometers use a vertical light path for through-the-plate reading. One result is that the length of the light path is not fixed by cuvette thickness, so that the absorbance varies with the volume of solution contained in each well. For a prototype of one through-the-plate photometer (Titertek Multiskan; Flow Laboratories, Inc., McLean, Va.), Ruitenberg et al. (5) evaluated intrarun imprecision and concluded that photometric inaccuracy is probably minor in comparison with biological variability and dispensing error in the performance of ELISA. We expanded these studies and evaluated the linearity of instrumental reading and the reproducibility of measurements within the same day and between 2 consecutive days for two commercially available photometers, Titertek Multiskan (Flow Laboratories) and Microelisa Autoreader (Dynatech Laboratories, Inc., Alexandria, Va.).

\section{MATERIALS AND METHODS}

Photometers. The Titertek Multiskan was equipped with 405-, 414-, 450-, and 492-nm filters with a specified half-band pass of 8 to $12 \mathrm{~nm}$. We estimated the half-band pass of the $405-\mathrm{nm}$ filter to be $10 \mathrm{~nm}$. The Microelisa Autoreader MR580 was equipped with 410-, 455-, 490-, and 570-nm filters; the half-band pass of these filters is specified as $10 \mathrm{~nm}$ but could not be

† Present address: General Hospital Pathologists, Ltd., General Hospital of Virginia Beach, Virginia Beach, VA 23454. assessed by us because of the design of the instrument Both photometers make an automatic background absorbance correction by means of a selected cup (Microelisa Autoreader) or a selected column of cups (Titertek Multiskan) filled with an appropriate buffer solution.

Test chromophore solution. A conventional chromogenic solution commonly used in ELISA (8) was prepared by incubating the enzyme alkaline phosphatase (from calf intestine, type VII; Sigma Chemical Co., St. Louis, Mo.) with its substrate, $p$-nitrophenylphosphate disodium (Sigma 104 phosphatase substrate tablets) in diethanolamine buffer (pH 9.8) at room temperature for $30 \mathrm{~min}$. The reaction was then stopped by the addition of $3 \mathrm{~N} \mathrm{NaOH}$. This chromophore solution was diluted with diethanolamine buffer to produce test solutions in the range of 0.050 to 1.400 absorbance units. The absorbance of the test solution at 405 and $410 \mathrm{~nm}$ was checked by repeated measurements with a calibrated dual-beam spectrophotometer (Beckman model 25; Beckman Instruments, Inc., Palo Alto, Calif.) before and after assays with each of the two photometers.

Microdispenser. The test solution was dispensed into the cups of microplates with a calibrated micropipette (Gilson Pipetman; Rainin Instrument Co. Inc., Woburn, Mass.). The coefficient of variation of this micropipette was less than $1 \%$.

Microplates. Flat-bottomed polystyrene microplates (Linbro EIA microplates; Flow Laboratories) with 12 columns of eight wells each were used.

Experimental designs. (i) Linearity of instrumental reading. (a) For each microplate, the first column of cups was filled with buffer, and columns 2 through 6 were filled with $200 \mu \mathrm{l}$ of test solution containing increasing amounts of chromophore. (b) For each microplate, the first column of cups was filled with buffer, and columns 2 through 6 were filled with 50 , $100,150,200$, and $250 \mu \mathrm{l}$ of a test solution containing a constant concentration of chromophore.

(ii) Effect of adding solvent to a determined amount of chromophore. To assess the effect of dispensing error in adding a solvent to a determined amount of chromophore, the first column of cups of a microplate was 


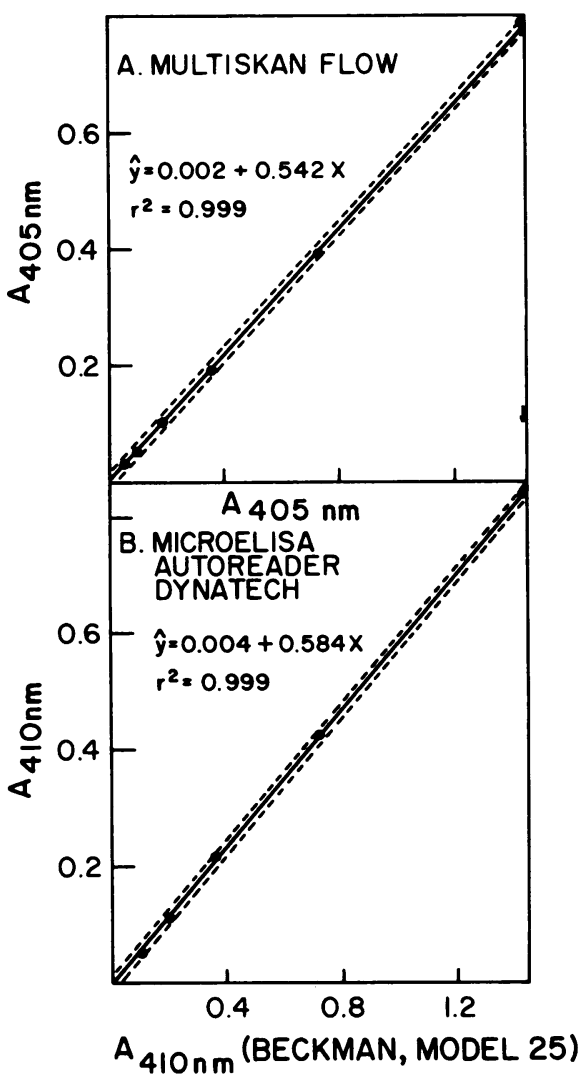

FIG. 1. Comparison of the Multiskan and Microelisa Autoreader with a dual-beam spectrophotometer. Abscissas: Absorbances at $405 \mathrm{~nm}$ (A) and 410 nm (B) determined with a Beckman model 25 spectrophotometer for $200 \mu$ l of solutions containing increasing amounts of chromophore. Ordinates: Absorbance of the same solutions determined at $405 \mathrm{~nm}$ with the Multiskan (A) and $410 \mathrm{~nm}$ with the Microelisa Autoreader (B). (-) Least-squares regression lines; (O) means of eight independent measurements; (---) $95 \%$ prediction intervals.

filled with $50 \mu \mathrm{l}$ of buffer, and columns 2 through 5 were filled with $50 \mu \mathrm{l}$ of chromophore solution. Then to columns 3 through 5 were added 50,100 , and $150 \mu$ l of buffer solution, respectively.

(iii) Intrarun imprecision. The intrarun imprecision of instrumental readings was assessed by performing eight repeated measurements of $200 \mu l$ of solutions with absorbance readings in the range of 0.050 to 0.850 absorbance unit.

(iv) Imprecision intraday and between days. The first column of a microplate was filled with $200 \mu$ l of buffer solution. Columns 2 through 12 were filled with $200 \mu l$ of test solution. Groups of independent measurements were taken the same day or over 2 consecutive days. To minimize evaporation, the plates were sealed and kept in a refrigerator at $4^{\circ} \mathrm{C}$.

Statistical analysis. Least-squares regression analysis (3) and one- and two-way analyses of variance (ANOVA) (3) were employed to study linearity and reproducibility of instrumental measurements, respec- tively. Data were analyzed by using the Statistical Package for the Social Sciences (4) or the Minitab Statistical Package (6), which were available to us through the University of North Carolina and the North Carolina Memorial Hospital computer systems. The graphical display of means comparisons was constructed by the method of Andrews et al. (1).

\section{RESULTS}

Since both of these photometers use a vertical light path for through-the-plate reading, the linearity of instrumental reading was verified by regression analysis of the absorbance curves generated by either increasing the amounts of chromophore in a fixed volume of test solution or increasing the volume of test solution containing a fixed concentration of chromophore. Figure 1 shows the linearity of readings when increasing amounts of chromophore were present in a fixed volume of test solution. Readings obtained with a dual-beam spectrophotometer were used for comparison. For the Titertek Multiskan, the regression equation was $y=$

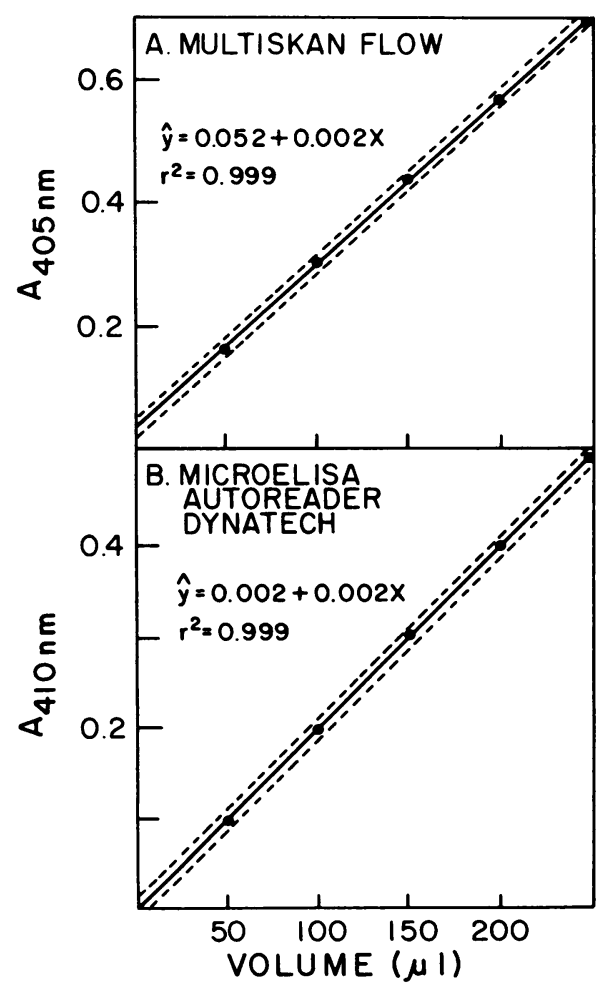

FIG. 2. Linearity of readings with increasing volumes of the same chromophore solution. Abscissas: Volumes of chromophore solution. Ordinates: Absorbances determined at $405 \mathrm{~nm}$ with the Multiskan (A) and at $410 \mathrm{~nm}$ with the Microelisa Autoreader (B). (-) Least-squares regression lines; $(O)$ means of eight independent measurements; (---) 95\% prediction intervals. 
TABLE 1. Effect of diluting a constant amount of chromophore: ANOVA results for the Microelisa Autoreader and Titertek Multiskan

\begin{tabular}{llrcccc}
\hline \multicolumn{1}{c}{ Photometer } & Source & df & $\begin{array}{l}\text { Sum of } \\
\text { squares }\end{array}$ & $\begin{array}{l}\text { Mean sum } \\
\text { of squares }\end{array}$ & $F$ ratio & $F$ probability \\
\hline Microelisa Autoreader & $\begin{array}{l}\text { Between } \\
\text { Within }\end{array}$ & 3 & 0.0001361 & 0.0000454 & 0.76 & $P=0.526$ \\
& 28 & 0.0016617 & 0.0000593 & & $P=0.029$ \\
Titertek Multiskan & Between & 3 & 0.001839 & 0.000613 & & 3.49 \\
& Within & 28 & 0.004916 & 0.000176 & \\
\hline
\end{tabular}

$0.002+0.542 x$, and the coefficient of determination, $r^{2}$, was 0.999 . For the Microelisa Autoreader, the regression equation was $y=0.004+$ $0.584 x$, and $r^{2}$ was 0.999 . The large coefficient of determination indicates a strong linear relationship between readings with a dual-beam spectrophotometer and with each of the two photometers. It was apparent by visual inspection that both regression lines were linear; this was confirmed by the test for lack of fit $(P<0.05)$ (3). The dashed lines delimit the $95 \%$ prediction interval, which was narrow and was about $\mathbf{0 . 0 1 0}$ absorbance unit over the range of measurements. These data indicate that the spread around the regression line was minimal, since for each level of $x, 95 \%$ of the observed values of $y$ fell within an interval of 0.020 absorbance unit.

The linearity of instrumental reading was also checked against volumetric increments of the same chromophore solution (Fig. 2). For both photometers, least-squares analysis generated straight regression lines with narrow $95 \%$ prediction intervals of about $\mathbf{0 . 0 1 5}$ absorbance unit and $r^{2}=0.999$. These results confirmed the linearity of instrumental readings and indicated that, as expected, these photometers differ from conventional photometers in that the volumetric error in dispensing a chromophore solution affects the overall error. The slopes of both regression lines indicate that a volumetric increment of $1 \mu l$ determined a photometric increment of 0.002 absorbance unit.

Conversely, for a through-the-plate-reading photometer, the volume in which a given amount of chromophore is diluted should not affect the mean absorbance measurements. We verified this hypothesis under extreme conditions. To four columns of eight wells of a microplate containing $50 \mu \mathrm{l}$ of chromophore in diethanolamine buffer we added 50,100 , or $150 \mu$ l of the same buffer or no buffer at all. The hypothesis of equality of mean absorbances among columns was then tested with one-way ANOVA. The ANOVA summary tables for the Titertek Multiskan and Microelisa Autoreader are shown in Table 1. For both photometers, the sums of squares (estimates of the variance) between and within groups (columns) were very small. For the Titertek Multiskan, the $F$ ratio is $3.49(P=$
0.029). For the Microelisa Autoreader, the $F$ ratio is $0.76(P=0.526)$. The results indicated that dilution of a given amount of chromophore in a nonabsorbing solution did not significantly affect the mean absorbance measurements.

The imprecision of instrumental measurements was assessed by comparing the means of groups of measurements taken either in the same run, in different runs the same day, or over 2 consecutive days. Table 2 shows the mean, standard deviation, and coefficient of variation (CV) for groups of measurements taken from the same microplate. The $\mathrm{CV}$ for both instruments was less than $12 \%$, even for the smallest absorbance value in the range studied. The $C V$ values obtained with the Microelisa Autoreader were somewhat smaller than those with the Titertek Multiskan.

The one-way ANOVA for the means of groups of eight measurements taken on the same day with the Microelisa Autoreader did not reveal statistically significant differences between these means $(P=0.468)$. Similarly, statistically significant differences were not found between means of groups of measurements performed over 2 consecutive days $(P=0.779)$.

Since the light beam for the Titertek Multiskan is divided into eight independent pathways,

TABLE 2. Intrarun imprecision of photometer measurements from a single microplate

\begin{tabular}{llcr}
\hline \multirow{2}{*}{$\begin{array}{l}\text { Photometer } \\
\text { group }\end{array}$} & \multicolumn{3}{c}{ Intrarun imprecision } \\
\cline { 2 - 4 } & Mean & SD & CV \\
\hline Microelisa & & & \\
Autoreader & & & \\
1 & 0.052 & 0.006 & 11.5 \\
2 & 0.106 & 0.004 & 3.8 \\
3 & 0.217 & 0.002 & 0.9 \\
4 & 0.430 & 0.002 & 0.5 \\
5 & 0.844 & 0.007 & 0.8
\end{tabular}

Titertek

\begin{tabular}{lrrr} 
Multiskan & & & \\
1 & 0.061 & 0.007 & 11.5 \\
2 & 0.108 & 0.010 & 9.2 \\
3 & 0.210 & 0.008 & 3.8 \\
4 & 0.413 & 0.008 & 1.9 \\
5 & 0.805 & 0.025 & 3.1 \\
\hline
\end{tabular}


TABLE 3. Titertek Multiskan: imprecision of same-day results two-way ANOVA between columns and rows (/)

\begin{tabular}{lrcrrr}
\hline Source & df & $\begin{array}{c}\text { Sum of } \\
\text { squares }\end{array}$ & $\begin{array}{c}\text { Mean sum of } \\
\text { squares }\end{array}$ & $F$ ratio & $F$ probability \\
\hline Rows & 7 & $\mathbf{0 . 0 0 0 8 3 9 0 9}$ & 0.00011987 & 13.26 & $P<0.0001$ \\
Columns & 10 & 0.00017861 & 0.00001786 & 1.98 & $P=0.0491$ \\
Error & 70 & 0.00063266 & 0.00000904 & & \\
\hline
\end{tabular}

which are aligned with corresponding rows of cups of a microplate, the imprecision was assessed by simultaneously testing the equality of mean measurements for columns and rows by two-way ANOVA (Table 3). There were no statistically significant differences between columns $(P=0.0491)$, but the differences between rows (eight separate light paths) were statistically significant $(P<0.0001)$. This large $F$ ratio is explained by the very small mean sum of squares error (0.00000904). This statistic is an unbiased estimate of the variance of the entire population of wells and was one order of magnitude smaller than the mean sum of squares between rows $(0.00011987)$. Thus, it was the very precision of the measurements which allowed a small systematic error between rows to be detected as statistically significant. The actual range of mean readings for rows was very narrow (0.557 to 0.565 absorbance unit) (Fig. 3), and there was partial overlapping of $95 \%$ confidence intervals (bars). Differences on the order

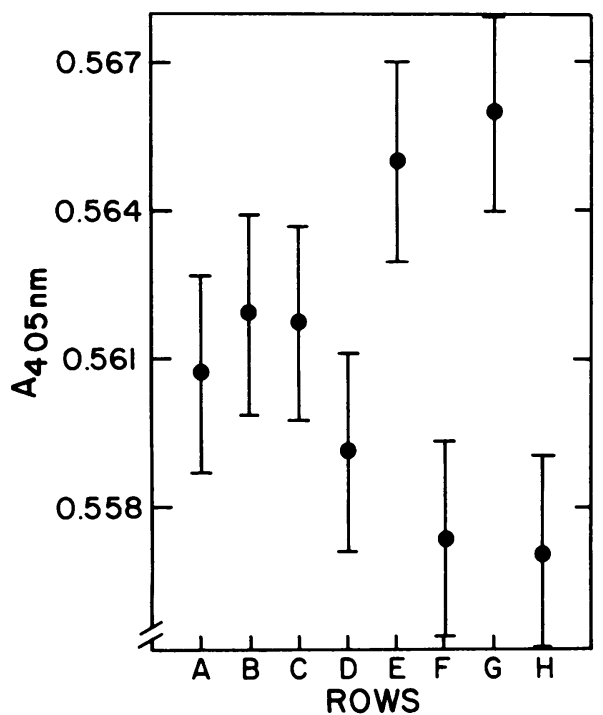

FIG. 3. Imprecision of the Multiskan readings between rows (eight separate light paths). All wells contained $200 \mu \mathrm{l}$ of the same chromophore solution. Abscissa: Plate coordinates for the rows. Ordinate: Absorbances at $405 \mathrm{~nm}$. The means of 11 measurements are indicated as dots and their $95 \%$ confidence intervals as bars. of thousandths of an absorbance unit are of no practical significance in results of ELISA, in which the inherent variability is much greater (7).

The means of 11 groups of eight measurements taken on 2 consecutive days were compared (Fig. 4). The differences within days were minimal, and the $95 \%$ confidence intervals (bars) were largely overlapping. The absorbance readings of day 2 were consistently greater than those of day 1 . The one-way ANOVA gave an $F$ ratio of $5.02(P<0.0001)$. However, again the pooled standard deviation was very small $(0.004$ absorbance unit), and the range of the means was narrow (0.550 to 0.561 absorbance unit). In this case also, very small differences were detectable as statistically significant because the overall variability of repeated readings was very small. Differences of this magnitude in ELISA results are of minimal practical significance. Further studies to determine the relative importance of the various components contributing to the sum of square errors for same-day and 2-day assays were not performed. However, the results of the dilution experiment indicated that evaporation of the solvent would have a trivial effect.

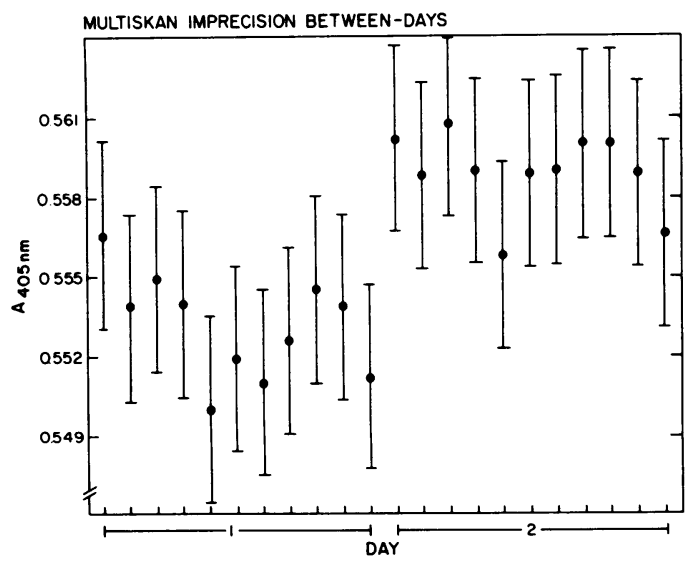

FIG. 4. Imprecision of the Multiskan over 2 days. All wells contained $200 \mu$ l of the same chromophore solution. Abscissa: 22 groups of measurements taken on 2 consecutive days. Ordinate: Absorbances determined at $405 \mathrm{~nm}$. The means of five repeated measurements are represented as dots and their $95 \%$ confidence intervals as bars. 


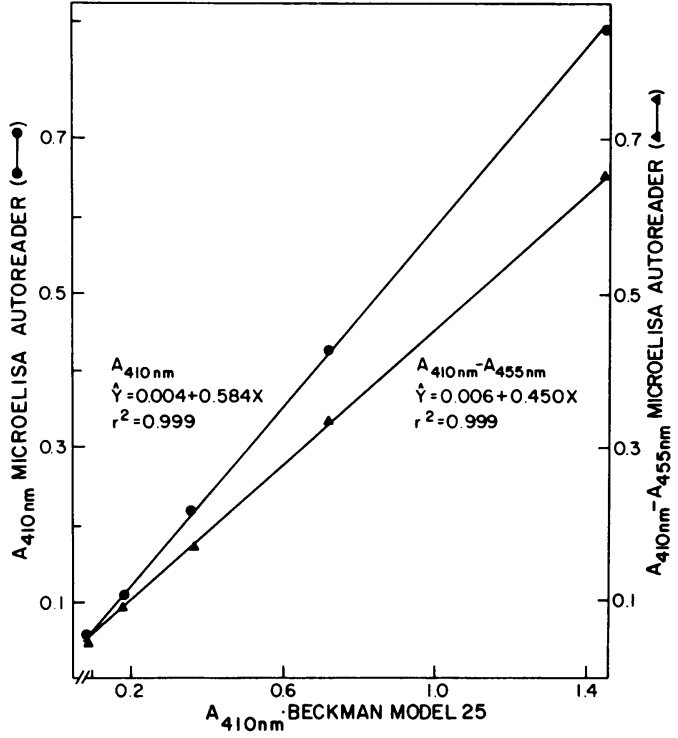

FIG. 5. Microelisa Autoreader, comparison of absorbance at $410 \mathrm{~nm}$ with that at $410-455 \mathrm{~nm}$ (dualwavelength mode of operation). Wells contained 200 $\mu l$ of solution with increasing amounts of chromophore. Abscissa: Absorbance determined with a Beckman model 25 spectrophotometer. Ordinates: Absorbance determined with the Microelisa Autoreader operating either in the single-wavelength mode (wavelength, $410 \mathrm{~nm}$ ) or in the dual-wavelength mode (wavelengths, 410 and $455 \mathrm{~nm}$ ).

The Microelisa Autoreader, as a means of correcting for scratches or other artifacts on the microplates, can compare readings at two wavelengths selected from the four standard filters supplied with the instrument. One of these wavelengths is selected to be near the absorption maximum of the chromophore used, and the other is selected to serve as a reference wavelength. If the reference wavelength is absorbed to some extent by the chromophore, the dualwavelength mode gives absorbance values which are smaller than those measured with the one-wavelength mode. Figure 5 shows this effect by comparing the regression lines obtained for the same chromophore solutions at the wavelength of $410 \mathrm{~nm}$ (this is the regression line shown in Fig. 1B) and at the wavelength of 410 - $455 \mathrm{~nm}$ (dual-wavelength mode). The two regression lines were significantly different by visual inspection, and this difference was confirmed by the statistical test for equality of regression lines $(P<0.0001)(3)$.

\section{DISCUSSION}

These studies indicated that for these two commercially available through-the-plate-reading photometers, linearity in measuring absor- bance of solutions with increasing amounts of chromophore and same-day and 2-day reproducibility were satisfactory.

The imprecision of instrumental readings was very small, on the order of thousandths of a unit over a range of 0.050 to 1.400 absorbance units. These estimates are similar to those reported by Ruitenberg et al. (5). As might be expected, the $\mathrm{CV}$ for both instruments was smaller for readings of at least 0.2 absorbance unit than for more dilute solutions. Therefore, the $\mathrm{CV}$ can be minimized by designing ELISA tests to give absorbance values greater than 0.2 for samples of interest.

The Titertek Multiskan showed statistically significant differences among mean absorbance readings of the eight individual light paths and between mean absorbance readings of the 2 consecutive days. However, these statistically significant differences were very small $(0.003$ and 0.004 absorbance unit, respectively) and of no practical clinical importance for the available ELISA tests, in which differences of $0.003 \mathrm{ab}$ sorbance unit are not critical in evaluating test results.

For both through-the-plate-reading photometers, there was a linear relationship between volumes of chromophore solution and absorbance readings, and the slope was 0.002 absorbance unit for both the Titertek Multiskan and the Microelisa Autoreader (Fig. 2). This clearly indicates, as do the results of Ruitenberg et al. (5), that volumetric inaccuracy in dispensing chromophore is a major source of error.

The volume in which a determined amount of chromophore was diluted did not affect the instrumental readings. Consequently, in ELISA, volumetric errors in adding buffer and inhibitor solutions do not affect the overall error of the assay.

To obtain comparable results of ELISA performed in laboratories with different throughthe-plate-reading photometers, the photometers should be calibrated against conventional spectrophotometers. Furthermore, for the Microelisa Autoreader, the performance of the dual-wavelength mode should be compared with that of the one-wavelength mode. It is important to note that readings taken in these two modes were not comparable, particularly for the larger amounts of chromophore (Fig. 5).

These studies were addressed to aspects of the evaluation of two commercially available through-the-plate-reading photometers. The results of this study confirm and extend those of Ruitenberg et al. (5), who studied aspects of the performance of a prototype of the Titertek Multiskan. For an exhaustive evaluation of the performance of spectrometers, the listing of specifcations proposed by the International 
Federation of Clinical Chemistry should be consulted (2).

\section{LITERATURE CITED}

1. Andrews, H. P., R. D. Snee, and M. H. Sarner. 1980. Graphical display of means. Am. Stat. 34:195-199.

2. Haeckel, R., C. H. Collonbel, T. D. Geary, F. L. Mitchell, R. G. Nedeau, and K. Okuda. 1980. Provisional guidelines (1979) for listing specifications of spectrometers in clinical chemistry. Clin. Chim. Acta 203:249-258.

3. Neter, J., and W. Wasserman. 1974. Applied linear statistical models. Richard D. Irwin, Inc., Homewood, Ill.

4. Nie, N. H., C. H. Hull, J. G. Jenkins, K. Steinbrenner, and D. H. Bent. 1970. Statistical package for the social sciences, 2nd ed. McGraw-Hill Book Co., New York.
5. Ruitenberg, E. J., V. M. Sekhuis, and B. J. M. Brosi. 1980. Some characteristics of a new multiple-channel photometer for through-the-plate reading of microplates to be used in enzyme-linked immunosorbent assay. J. Clin. Microbiol. 11:132-134.

6. Ryan, T. A., Jr., B. L. Joiner, and B. F. Ryan. 1976 Minitab: a student handbook. Duxbury Press, North Scituate, Mass.

7. Vejtorp, M. 1978. Enzyme-linked immunosorbent assay for determination of rubella IgG antibodies. Acta Pathol. Microbiol. Scand. Sect. B 86:387-392.

8. Voller, A., D. Bidwell, and A. Bartlett. 1980. Enzymelinked immunosorbent assay, p. 359-371. In N. R. Rose and H. Friedman (ed.), Manual of clinical immunology, 2nd ed. American Society for Microbiology, Washington, D.C. 